\title{
Thermomechanical deformation of the orthotropic shell taking into account the deformation anisotropy
}

\author{
Aleksandr Treshchev*1, Mariya Lapshina ${ }^{1}$, and Yulia Zavyalova ${ }^{10000-0002-8394-8037]}$ \\ ${ }^{1}$ Tula State University, 300012 Tula, Russia
}

\begin{abstract}
A variant of the rotation shell in the particular form of a closed circular cylindrical shell, which is often used in the design practice of civil, power and other industrial structures, is considered. The specificity of the considered shell lies in the features of its material, which has a manifestation of dual anisotropy. In particular, this material is orthotropic in structure, and the nature of deformation shows the dependence of stiffness and strength on the type of stress state. The loading of the shell is assumed to be axisymmetric, taking into account the influence of a medium with variable thermal parameters. The temperature difference between the shell surfaces is taken into account here. The statement of the general thermomechanical problem is carried out in an unrelated form, taking into account a certain independence of the problems of thermodynamics and mechanics. Taking into account the limitations of the classical thermomechanical theories of shells made of materials with dual anisotropy and the fact that the known models for such materials have significant drawbacks, the authors used a variant of the normalized stress space. Differential equations of thermoelasticity for a cylindrical shell are obtained, taking into account the complicated thermomechanical properties of its material. Particular solutions with the features of the results of calculating the shell states are illustrated, and their analysis is carried out.
\end{abstract}

Keywords. Orthotropic structure, induced anisotropy, thermomechanics, unbound thermoelasticity problem, cylindrical shell.

\section{Introduction}

In modern construction, high-strength composite materials are widely used, the basis of which is polymers with directional or chaotic reinforcement with basalt and «glass fibers», the thermomechanical properties of which differ significantly from the common steel and reinforced concrete structures [1-7]. In particular, these materials can have structural anisotropy of different classes, and when they are deformed under load, they react differently to the magnitude and ratios of the resulting stresses, so that the compliance tensor is not invariant to the type of stress state. Such materials are commonly referred to as deformable structures with double anisotropy. In structurally anisotropic materials, the dependence on the directions is shown not only in the stiffness characteristics, but also in the thermal-physical ones [8-11]. The

${ }^{*}$ Corresponding author: taa58@yandex.ru 
most common in construction are structural materials with structural orthotropy of physical and mechanical properties. Traditional computational models of solid-state mechanics and structural mechanics, formally operating with the generalized Hooke's law or the relations of small elastic-plastic deformations of A.A. Ilyushin [12], lead to serious errors not only in the obtained quantitative characteristics of the stress-strain state of building structures, but also distort its qualitative picture. Such facts do not allow us to ensure the proper accuracy of design calculations and the reliability of design decisions. In the light of the above, for the calculation of building structures made of these materials, it is necessary to use proven theories and models, as much as possible confirmed by experimental data.

The change in the operating temperature of building structures based on materials with anisotropy of two kinds introduces even greater difficulties in mathematical models and methods of their calculation. The solution of thermoelasticity problems for structures made of materials with structural and deformation anisotropy is still episodic and does not allow us to develop methodological recommendations for the design of particularly thin-walled spatial structures. Since the materials under consideration have high elastic modulus and strength limits, they are widely used in the manufacture and construction of thin-walled shells, which, with a minimum thickness and total weight, have significant advantages in load-bearing capacity.

Below we consider a particular version of the shells of rotation, namely, a closed circular cylindrical shell, which is subjected to the most common axisymmetric load in the form of uniform pressure. Cylindrical boilers that are subject to steam pressure, similar tanks with a vertical or horizontal axis that are affected by the internal pressure of a liquid or gas, as well as pipelines with hydraulic pressure - these are exactly the objects of this kind of calculation tasks. It is obvious that the design of these structures, in addition to the internal pressure, is subject to temperature fluctuations during operation.

Almost all known orthotropic deformation materials exhibit the effect of the coupling of temperature and deformation fields only in a short period of the initial application of the temperature difference on opposite surfaces of structures, and the magnitude of this effect is quite small. After the appearance of a steady-state stationary temperature drop, the effect of the coupling of thermal and force fields disappears altogether [13, 14]. Therefore, the mechanism of heat transfer occurring in an unevenly heated structure due to contact with the environment allows us to neglect the influence of the stress state on the temperature distribution in it. In this regard, the study of the temperature field in the body of the structure corresponding to the specific conditions of heat transfer is carried out independently of its stress-strain state, that is, an unrelated problem of thermoelasticity is considered here.

\subsection{Problem statement}

In a cylindrical coordinate system, we consider a circular cylindrical shell made of an orthotropic deformation-anisotropic material $[1,2]$. The position of a specific point of the shell body is determined by Gaussian variables $\beta_{1}, \beta_{2}, \beta_{3}$. We assume that the coordinate axes coincide with the main axes of orthotropy for the following geometric parameters: length $L$, wall thickness $h$, radius of the average cylindrical surface $R$. One end of the shell is assumed $\beta_{l}=0$ to be rigidly pinched, and the opposite end is assumed $\beta_{l}=L$ to be free from anchoring and loading. The thermal state of the shell is such that at the initial moment of time, the temperature at any point of the shell is the same $-T_{0}$, and subsequently, a temperature difference is established such that the temperature on the inner surface $T_{1}$ and the outer surface arose $-T_{2}$. In the future, we assume that the temperature changes occur only over the thickness of the shell with the temperature difference $\Delta T$ being a function of the coordinate $\beta_{3}$. Force loading is carried out by a uniformly distributed load intensity $q_{3}$ from inside the shell (internal pressure). 
Let us assume a shell so thin that the application of the Kirchhoff-Love hypotheses was not in doubt, and a configuration such that it could be classified as flat, for which dependencies are applicable:

a) strains of the median surface:

$$
\varepsilon_{1}=u_{,_{1}}+0,5 \theta_{1}^{2} ; \quad \varepsilon_{2}=v_{,_{1}}+k w+0,5 \theta_{2}^{2} ; \quad \gamma=v_{,_{1}}+u,_{2}+\theta_{1} \theta_{2}
$$

where $u, v, w$ is the displacement in the median surface (axial, tangential, and radial); $k=1 / R$ - the main curvature in the undeformed state; $\theta_{1}=-w_{1} ; \theta_{2}=-w_{2}+k v$;

b) curvature and torsion of the median surface:

$$
\chi_{1}=-w_{11} ; \chi_{2}=-w,,_{22} ; \tau=-w,,_{12} ;
$$

c) complete deformations at any point of the shell with a coordinate $\beta_{3}$ relative to the median surface:

$$
e_{11}=\varepsilon_{1}+\beta_{3} \chi_{1} ; \quad e_{22}=\varepsilon_{2}+\beta_{3} \chi_{2} ; \gamma_{12}=\gamma+2 \beta_{3} \tau \text {. }
$$

In contrast to [15-18], the equations for the connection of strain and stress tensors are taken on the basis of the strain potential, which is presented in the framework of the normalized stress tensor space, the most reasonable and theoretically consistent, which is presented in detail in the studies [19-21]. Obviously, taking into account the influence of the temperature difference, the Kirchhoff-Love hypotheses reduce these equations in the main axes of orthotropy to the form:

$$
\begin{aligned}
& e_{11}=C_{111} \sigma_{11}+C_{1122} \sigma_{22}+\alpha_{1 T} \Delta T ; \\
& e_{22}=C_{1122} \sigma_{11}+C_{2222} \sigma_{22}+\alpha_{2 T} \Delta T ; \\
& \gamma_{12}=2 e_{12}=C_{1212} \tau_{12},
\end{aligned}
$$

where $C_{1111}=\left(A_{1111}+B_{1111} \alpha_{11}\right)+0,5\left[B_{1111} \alpha_{11}\left(1-\alpha_{11}^{2}\right)-B_{2222} \alpha_{22}^{3}-B_{1212} \alpha_{12}^{3}\right]+$

$$
\begin{aligned}
& +B_{1122} \alpha_{22}\left(1-\alpha_{11}^{2}-\alpha_{11} \alpha_{22}\right) ; C_{2222}=\left(A_{2222}+B_{2222} \alpha_{22}\right)+ \\
& +0,5\left[B_{2222} \alpha_{22}\left(1-\alpha_{22}^{2}\right)-B_{1111} \alpha_{11}^{3}-B_{1212} \alpha_{12}^{3}\right]+B_{1122} \alpha_{11}\left(1-\alpha_{22}^{2}-\alpha_{11} \alpha_{22}\right) ; \\
& C_{1212}=A_{1212}-\left(B_{1111} \alpha_{11}^{3}+B_{2222} \alpha_{22}^{3}\right)-2 B_{1122} \alpha_{11} \alpha_{22}\left(\alpha_{11}+\alpha_{22}\right) ; \\
& C_{1122}=A_{1122}+B_{1122}\left(\alpha_{11}+\alpha_{22}\right) ;
\end{aligned}
$$

$A_{i j k m}, B_{i j k m}$ - potential constants defined in terms of elastic modulus, coefficients of transverse deformation of the material in the main axes of orthotropy for tension, compression, and shear modulus; $\alpha_{i j}=\sigma_{i j} / S$ - components of the normalized stress tensor; $S=\sqrt{\sigma_{i j} \sigma_{i j}}=\sqrt{\sigma_{11}^{2}+\sigma_{22}^{2}+2 \tau_{12}^{2}}-$ stress tensor modulus; $\alpha_{1 T}, \alpha_{2 T}-$ coefficients of linear temperature expansion in the main axes of orthotropy.

After testing the standard samples for axial tension and compression in the main axes of orthotropy and for shear in the main planes [20,21], we obtain the values of the constants of the equations (4):

$$
\begin{gathered}
A_{k k k k}=\left(1 / E_{k}^{+}+1 / E_{k}^{-}\right) / 2 ; B_{k k k k}=\left(1 / E_{k}^{+}-1 / E_{k}^{-}\right) / 2 ; A_{i j i j}=1 / G_{i j} ; \\
A_{i i j j}=-\left(v_{i j}{ }^{+} / E_{j}^{+}+v_{i j}^{-} / E_{j}^{-}\right) / 2 ; B_{i j j}=-\left(v_{i j}^{+} / E_{k}{ }^{+}-v_{i j} / E_{j}^{-}\right) / 2,
\end{gathered}
$$

where $v_{i j}{ }^{+} / E_{j}^{+}=v_{j i}{ }^{+} / E_{i}^{+} ; v_{i j}^{-} / E_{j}^{-}=v_{j i}{ }^{-} / E_{i}^{-} ; E_{k}{ }^{ \pm}, v_{i j}{ }^{ \pm}$- elastic modulus and transverse strain coefficients for axial tension and compression in the main axes of orthotropy; $G_{i j}$ - the shear modulus in the main plane of the orthotropy; $i, j, k=1.2$.

\section{Materials and methods}

\subsection{System of resolving differential equations}

To formulate the resolving equations for calculating the shell, we invert the equations of state

(4) with respect to the stresses:

$$
\sigma_{11}=D_{11} e_{11}+D_{12} e_{12}-R_{11}-\varphi_{1 T} ; \tau_{12}=D_{66} e_{12}-R_{12} ; \sigma_{22}=D_{12} e_{11}+D_{22} e_{12}-R_{22}-\varphi_{2 T},
$$




$$
\begin{aligned}
& \text { where } D_{11}=C_{2222} / \Delta ; D_{12}=-C_{1122} / \Delta ; D_{22}=C_{1111} / \Delta ; D_{66}=1 / C_{1212} ; R_{11}=\left(A_{2222} T_{11}-A_{1111} T_{22}\right) / \Delta ; \\
& \quad R_{22}=\left(A_{1111} T_{22}-A_{1122} T_{11}\right) / \Delta ; R_{12}=T_{12} / A_{1212} ; \\
& T_{11}=\left\{B_{1111} \alpha_{11}+0,5\left[B_{111} \alpha_{11}\left(1-\alpha_{11}^{2}\right)-B_{2222} \alpha_{22}^{3}-B_{1212} \alpha_{12}^{3}\right]+\right. \\
& \left.+B_{1122} \alpha_{22}\left(1-\alpha_{11}^{2}-\alpha_{11} \alpha_{22}\right)\right\} \sigma_{11}+B_{1122}\left(\alpha_{11}+\alpha_{22}\right) \sigma_{22} ; \\
& T_{12}=-\left[\left(B_{1111} \alpha_{11}^{3}+B_{2222} \alpha_{22}^{3}\right)-2 B_{1122} \alpha_{11} \alpha_{22}\left(\alpha_{11}+\alpha_{22}\right)\right] \tau_{12} ; \\
& T_{22}=B_{1122}\left(\alpha_{11}+\alpha_{22}\right) \sigma_{11}+\left\{B_{2222} \alpha_{22}++0,5\left[B_{2222} \alpha_{22}\left(1-\alpha_{22}^{2}\right)-B_{1111} \alpha_{11}^{3}-\right.\right. \\
& \left.\left.-B_{1212} \alpha_{12}^{3}\right]+B_{1122} \alpha_{11}\left(1-\alpha_{22}^{2}-\alpha_{11} \alpha_{22}\right)\right\} \sigma_{22} ; \varphi_{1 T}=D_{11} \alpha_{1 T} \Delta T+D_{12} \alpha_{2 T} \Delta T ; \\
& \varphi_{2 T}=D_{12} \alpha_{1 T} \Delta T+D_{22} \alpha_{2 T} \Delta T ; \Delta=A_{1111} A_{2222}-A_{1122}^{2} .
\end{aligned}
$$

It is known that the equilibrium equations and Cauchy relations do not depend on the physical nature of the material, so for shells made of materials with anisotropy of two kinds, these classes of dependencies do not change. Therefore, the equations of equilibrium and continuity of deformations retain their form as for the general nonlinear theory of anisotropic shells [22]. In this case, all internal torques and forces are brought to the median surface of the cylindrical shell at $\beta_{3}=0$. Taking into account that the shell is loaded with an internal uniform pressure in the absence of torques $\left(\tau_{12}=e_{12}=0\right)$ and $\beta_{3} k \ll 1$, the equations of equilibrium and continuity of deformations are simplified, transforming to the form:

$$
N_{l^{\prime} l}=0 ; M_{l^{\prime},{ }^{-}} Q_{1-}-N_{l} \theta_{l}=0 ; Q_{l^{\prime} l}-k N_{2}+q_{3}=0 ; k \chi_{l}+\varepsilon_{2^{\prime}{ }_{11}}=0
$$

and from the geometric relations, the following are preserved:

$$
\varepsilon_{1}=u_{1}+0.5 \theta_{1}^{2} ; \varepsilon_{2}=k w ; \chi_{1}=-w \cdot{ }_{11} ; e_{11}=\varepsilon_{1}+\beta_{3} \chi_{1} ; e_{22}=\varepsilon_{2},
$$

where $N_{k}$ - longitudinal forces in the middle surface of the shell; $Q_{1}$ - lateral force; $M_{1}-$ bending moment:

$$
N_{k}=\int_{-h / 2}^{h / 2} \sigma_{k k} d \beta_{3} ;(\mathrm{k}=1.2) ; M_{11}=\int_{-h / 2}^{h / 2} \sigma_{11} \beta_{3} d \beta_{3} .
$$

Integrating equations (5) over the thickness of the shell according to the rules (8), introducing the results into static equations (6), geometric dependencies (7) continuity ratio (6), while performing the appropriate recombination, we obtain two mixed differential equations:

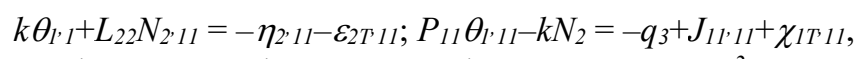

where $L_{11}=K_{22} / \Delta ; L_{22}=K_{11} / \Delta ; L_{12}=-K_{12} / \Delta ; \Delta=K_{11} K_{22}-K_{12}^{2} ; K_{i j}=D_{i j} h ; P_{i j}=D_{i j} h^{3} / 12$; $\eta_{1}=L_{11} I_{11}+L_{12} I_{22} ; \eta_{2}=L_{12} I_{11}+L_{22} I_{22} ; I_{i j}=\int_{-h / 2}^{h / 2} R_{i j} d \beta_{3} ; J_{i j}=\int_{-h / 2}^{h / 2} R_{i j} \beta_{3} d \beta_{3} ; \eta_{i T}=\int_{-h / 2}^{h / 2} \varphi_{i T} d \beta_{3}$ $; \chi_{i T}=\int_{-h / 2}^{h / 2} \varphi_{i T} \beta_{3} d \beta_{3} ; \varepsilon_{1 T}=L_{11} \eta_{1 T}+L_{12} \eta_{2 T} ; \varepsilon_{2 T}=L_{12} \eta_{1 T}+L_{22} \eta_{2 T}$.

Taking into account that, according to the condition of the task, the end of the shell at is $\beta_{l}=L$ pinched, and the end at is $\beta_{l}=0$ completely free, the boundary conditions for the latter have the form:

$$
\theta_{1}=0 ; \varepsilon_{1}=0 ; \varepsilon_{2}=0 \Rightarrow N_{2}=-\frac{\eta_{2}+\varepsilon_{2 T}}{L_{22}}
$$

\subsection{Temperature component of the task}

The temperature difference between the inner and outer surfaces of the shell requires a significant adjustment of the results of the calculation of its stress-strain state [23]. The determination of the temperature components in the problem under consideration requires an 
independent consideration of the process of heat transfer through the shell. This process is uniquely determined by the well-known equation of thermal conductivity:

$$
T_{t}=a_{1} \cdot T_{11}+a_{2} \cdot T_{22}+a_{3} \cdot T_{33},
$$

where $a_{1}, a_{2}, a_{3}$ - thermal conductivity coefficients that determine the thermal inertia of the material in the characteristic directions; $t$ - time parameter; $T$ - parameter of the temperature field.

Let us consider a stationary process with a one-dimensional propagation of the temperature field with a difference only over the thickness of the shell, when the temperature changes only along one coordinate $\beta_{3}$ orthogonal to the isothermal surfaces. Then, the heat equation is simplified and takes the form: $T_{t}=a_{3} T_{33}$, where $a_{3}=\lambda / c-$ the coefficient of thermal conductivity normal to the curved surface of the shell; $\lambda$-coefficient of thermal conductivity; $c$ - specific volumetric heat capacity of the material.

Due to the fact that the coefficient of thermal conductivity for the materials under study is quite large, for a thin-walled shell, the process of achieving a stationary equilibrium linear temperature distribution over its thickness occurs very quickly in a short time interval. Therefore, we can conclude that it is advisable to study the thermomechanical problem at the time of the equilibrium temperature distribution.

For the equilibrium temperature distribution over the shell thickness, the calculation of the temperature difference $\Delta T$ in the shell is determined by the linear distribution law:

$$
T\left(\beta_{3}\right)=\left(T_{2}-T_{1}\right) \beta_{3} / h+\left(T_{1}+T_{2}\right) / 2-T_{0} .
$$

For the temperature component of the problem, in addition to the conditions at the boundaries, initial conditions are required, when at the initial time at $t=0$ for any point of the shell body the temperature is the same and equal $T_{0}$, and the boundary conditions at the current time $t=t_{k}$ are assumed in accordance with the linear temperature distribution over the thickness $T_{1}$ and $T_{2}$, on the inner and outer surfaces, respectively.

Due to the significant nonlinearity of the two-fold problem under consideration, its solution was constructed in increments of the desired functions by the two-step method of successive perturbations of the parameters of V.V. Petrov [24, 25] in combination with a finite-difference approximation of increased accuracy [26], integration by the thickness method of Simpson, and by the load - Adams. In this case, the system of algebraic equations was solved by the Gauss method.

\section{Results and discussions}

\subsection{Results of solving the boundary value problem and their analysis}

We determine the stress-strain state of the shell, the design scheme of which is shown in Fig. 1. The main geometric parameters of the shell are taken as follows: length $L=2.0 \mathrm{~m}$; thickness $h=0.04 \mathrm{~m}$; diameter of the median surface $2 \mathrm{R}=0.8 \mathrm{~m}$. A variant of applying an internal pressure with an intensity of $q_{3}=8 \mathrm{MPa}$ is considered. The temperature medium transfers heat due to the thermal conductivity of the shell material so that a constant temperature $T_{l}=0^{\circ} \mathrm{C}$ is maintained inside the shell, and a constant temperature $T_{2}=+30^{\circ} \mathrm{C}$ is maintained outside at the initial temperature of the entire shell $T_{0}=0^{\circ} \mathrm{C}$.

The shell material is a three - reinforced woven polymer composite of the P36-50 brand [1], for which the main parameters are established: elastic modulus and transverse deformation coefficients along the main axes of orthotropy $-E_{1}^{+}=10.3 \mathrm{GPa}, E_{1}^{-}=11.77 \mathrm{GPa}, E_{2}{ }^{+}=17.6 \mathrm{GPa}$, $E_{2}^{-}=18.54 \mathrm{GPa}, v_{12}{ }^{+}=0.188, v_{12}{ }^{-}=0.125$; thermal expansion coefficients $\alpha_{1 T^{ \pm}}=33 \cdot 10^{-5 \circ} \mathrm{C}^{-1}$, $\alpha_{2 T^{ \pm}}=40 \cdot 10^{-50} \mathrm{C}^{-1}$, [2]. As can be seen, the degree of resistance in all directions is assumed to be minimal: $14.3 \%$ along the axis $\beta_{1}$ and $5.3 \%$ along the - axis $\beta_{2}$. This is an extremely minimal value of the difference in resistance with a minimum temperature difference. 

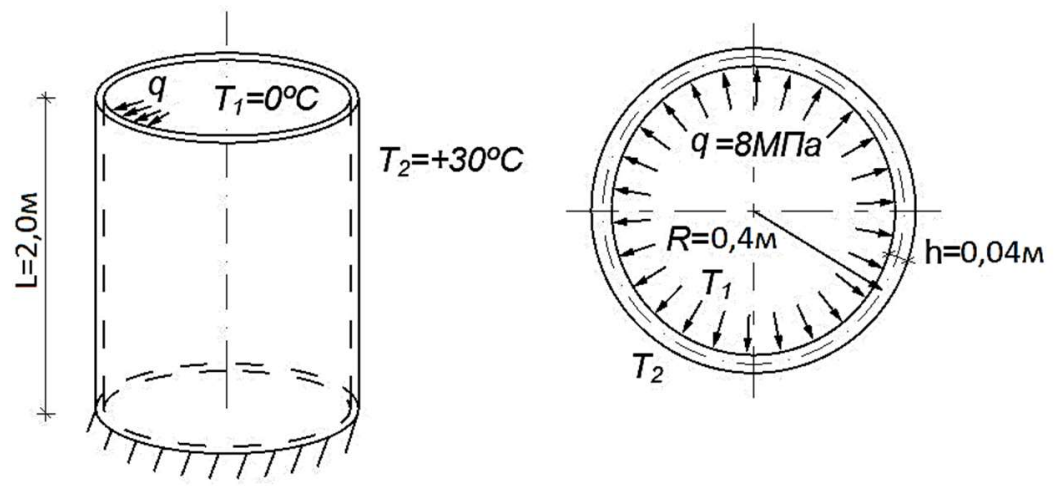

Fig. 1. Design scheme of the shell.

Fig. 2-4 show the main results of the calculation of the deformation of the shell, taking into account the accepted conditions. The error in determining the maximum deflections during loading with and without temperature exposure is $20.8 \%$.

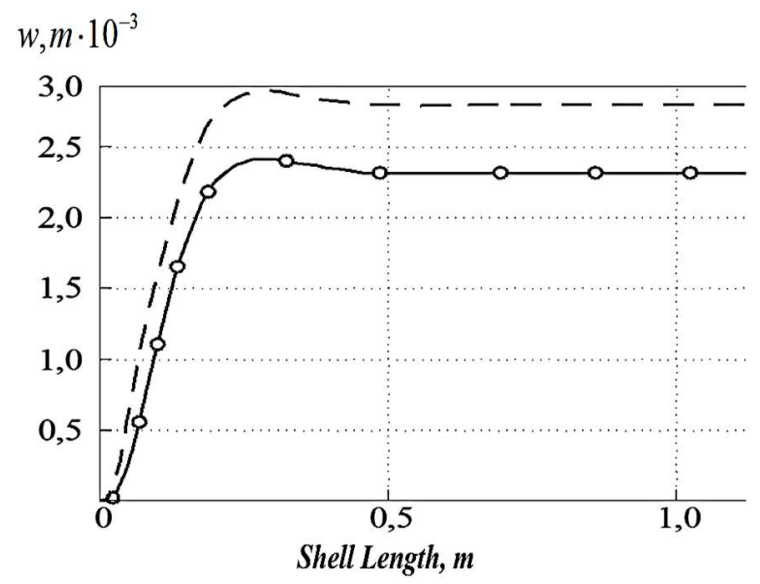

Fig. 2. Deflections of the shell.

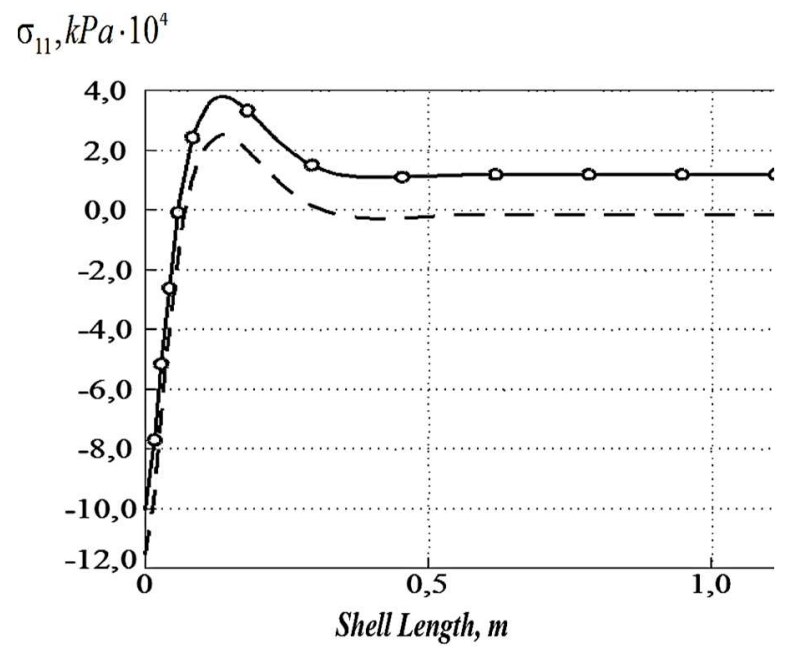

Fig. 3. Axial stresses. 


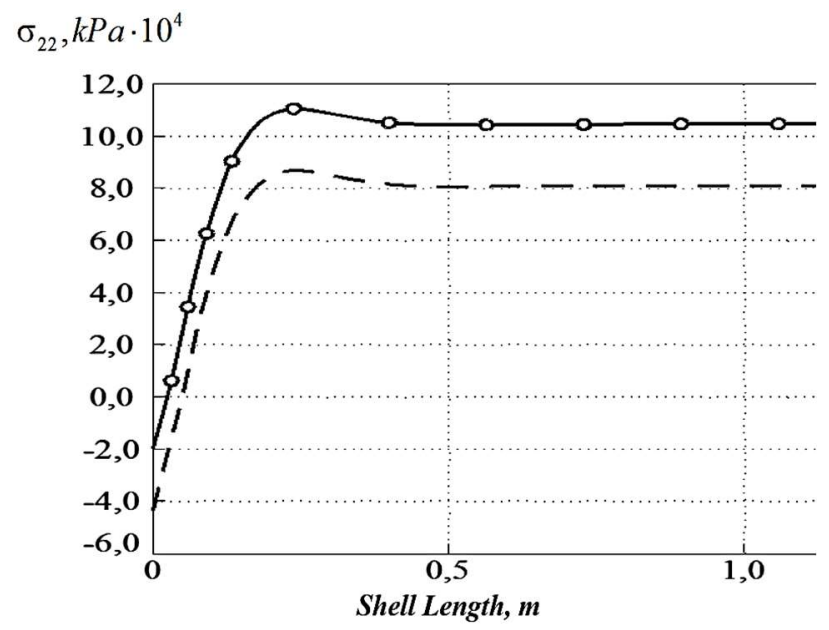

Fig. 4. Circumferential stresses.

The axial and circumferential stresses on the inner surface of the shell are completely identical without and under thermal influence, which is due to the coincidence of temperatures on this surface at any given time. The axial stresses on the outer surface in the seal without temperature loading are $15 \%$ less than in the case of it, but for the highest tensile stresses this difference reaches $34.6 \%$. Moreover, in the area of $0.125 L-L$, the effect of temperature changes the stress sign to the opposite.

The circumferential stresses from the outer surface of the shell, without taking into account the thermal effect, are 2 or more times lower compared to the calculation option when heated at the pinched end. The maximum circumferential stresses in the outer fibers of the shell when heated are $21.5 \%$ less than without it. If the shell material is a variant with large differences in the strain characteristics for tension and compression in the directions of the main axes of orthotropy, then the error of classical theories will significantly increase, it can reach $100 \%$ in some indicators, and sometimes even more [27].

\section{Conclusions}

The analysis of the calculation results shows that taking into account the heating process of cylindrical shells made of orthotropic materials with deformation anisotropy under temperature influences shows a cardinal discrepancy between the obtained results of general classical theories, which need to be modernized taking into account the universal equations of state (4). This is even shown when using simplified models [11, 15-18].

\section{References}

1. A.V. Rose, I.G. Zhigulin, M.N. Dushin. Three reinforced fabric materials, Mechanics of polymers 3, 471-476 (1970).

2. V.A. Kargin. Encyclopedia of polymer. M.: Sovetskaya Entsiklopediya 1, 1224 (1972).

3. E.V. Amelina. On nonlinear deformation of carbon fiber plastics: experiment, model, calculation, ICT SD RAS: Computational Technologies 20 5, 27-52 (2015).

4. R.A. Kayumov, S.A. Lukanin, V.N. Paimushin, S.A. Kholmogorov. Identification of mechanical characteristics of fiber-reinforced composites, Scientific notes of the Kazan University. Physical and mathematical sciences 157 4, 112-132 (2015). 
5. L.N. Shafigullin, A.A. Bobrishev, V.T. Erofeev, A.A. Treshchev, A.N. Shafigullina. Development of the recommendations on selection of glass-fiber reiforced polyurethanes for vehicle parts, International Journal of Applied Engineering Research 10 23, 43758$43762(2015)$.

6. A.A. Ysupova, R.T. Akhmetova, A.A. Treshchev, L.N. Shafigullin, A.V. Lakhno, A.A. Bobrishev. Sulfur composite technology from oil refinery waste, International Journal of Applied Engineering Research 11 Iss. 5 1, 3057-3061 (2016).

7. A.A. Ysupova, R.T. Akhmetova, A.A. Treshchev, A.A. Bobrishev, L.N. Shafigullin, G.R. Shayakhmetova. Production of Sulfur Composite Materials from Sulfur Containing Waste for Construction Applications, Research Journal of Pharmaceutical, Biological and Chemical Sciences 7 (4), 1411-1419 (2016).

8. P.E. Hart. The effect of pre-stressing on the thermal expansion and young's modulus of graphite, Carbon 10, 233-236 (1972).

9. Y.S. Hsu, C.W. Bert, J.N. Reddy. Thermoelasticity of Circular Cylindrical Shells Laminated of Bimodulus Composite Materials, Journal of Thermal Stresses 4 2, 155-177 (1981).

10. J.N. Reddy, C.W. Bert, Y.S. Hsu, V.C. Reddy. Thermal bending of think rectangular plates of bimodulis composite materials, Journal Mach. Eng. Sci. 22 6, 297-304 (1980).

11. A.A. Treschev, M.V. Spasskaya, M.B. Shereshevsky, A.A. Bobrishev, L.N. Shafigullin. The influence of temperature differences for the analysis of thin orthotropic cylindrical shell, Scientific Review Engineering and Environmental Sciences 26 4, 528-537 (2017).

12. A.A. Ilyushin. Plasticity. M.: Publishing House of the USSR Academy of Sciences, 271 (1963).

13. A.A. Treschev, M.Yu. Delyagin. Modeling of a shell made of isotropic multi-resistive graphite using bulk finite elements, taking into account the coupling of stresses and temperatures, Materials Physics and Mechanics 17 1, 59-70 (2013).

14. A.A. Treschev, M.Yu. Delyagin. Associated thermomechanical calculation of the composite graphite shell with consideration of substantially nonlinear resistance to different materials, Bulletin of the Volga State Technological University. Ser.: Materials. Structures. Technologies 3 (11), 91-100 (2019).

15. A.A. Treschev, V.G. Telichko, P.Yu. Khodorovich. Modeling of the stress-strain state of thick cylindrical shells made of materials with complicated properties, Materials Physics and Mechanics 21 1, 38-50 (2014).

16. M.V. Spasskaya, A.A. Treschev. Thermomechanical problem for a flat spherical shell made of a material with complicated properties, Construction mechanics and calculation of structures 5, 58-65 (2018).

17. A.A. Treschev, M.V. Spasskaya. Stress-strain state of a circular cylindrical shell made of a material with complicated properties, Materials Physics and Mechanics 29 1, 32-38. (2016).

18. L.N. Shafigullin, A.A. Treschev P.Yu. Hodorovich, V.T. Erofeev. The Stress-Strain State of Layered Orthotropic Conditional Half-Space Taking Into Account Different Resistance, Revista Publicando 413 (2), 109-127 (2017).

19. A.A. Treschev, A.A. Bobrishev, L.N. Shafigullin. Constitutive relations for isotropic materials allowing quasi-linear approximation of the deformation law, IOP Conference Series: Materials Science and Engineering 481, 012014. (2019). DOI: 10.1088/1757899X/481/1/012014.

20. A.A. Treschev, Yu.A. Monastyrev, V.D. Chibrikina, Yu.A. Zavyalova, M.A. Lapshina. Description of deformation of orthotropic materials with different resistance characteristics, Construction mechanics and structures 1 (20), 7-13 (2019). 
21. A.A. Treschev, Yu.A. Zavyalova, M.A. Lapshina. Variant of the deformation model of orthotropic composite materials, Expert: Theory and Practice (Scientific and Practical Journal) 3 (6), 62-68 (2020). DOI: 10.24411/2686-7818-2020-10027.

22. S.A. Ambartsumyan. General theory of anisotropic shells. M.: Nauka, 446 (1974).

23. A.D. Kovalenko. Thermoelasticity. Kiev: Vishcha shkola, 216 (1975).

24. V.V. Petrov, I.V. Krivoshein. Methods for calculating structures made of non-linearly deformable material. M.: Publishing House of the DIA, 208 (2009).

25. V.V. Petrov, I.V. Krivoshein. Inhomogeneous flat shells with two types of nonlinearities, ACADEMIA. Architecture and construction 1, 114-123 (2013).

26. P.M. Varvak, L.P. Varvak. The method of grids in problems of calculation of building structures. M.: Stroyizdat, 160 (1977).

27. A.A. Treschev, E.A. Zhurin. Bending of Ring Plates, Performed from an Orthotropic Nonlinear Differently Resistant Material, International Journal for Computational Civil and Structural Engineering 16, Iss. 1, 130-146 (2020). 